\title{
Front dynamics in the presence of spatiotemporal structured noises
}

\author{
Miguel A. Santos ${ }^{1, *}$ and J. M. Sancho ${ }^{2}$ \\ ${ }^{1}$ Departament d'Enginyeria Mecànica, Universitat Rovira i Virgili, Carretera Salou s/n, 43006 Tarragona, Spain \\ ${ }^{2}$ Departament d'Estructura i Constituents de la Matèria, Universitat de Barcelona, Avenida Diagonal 647, 08028 Barcelona, Spain
}

(Received 7 December 2000; published 27 June 2001)

\begin{abstract}
Front dynamics modeled by a reaction-diffusion equation are studied under the influence of spatiotemporal structured noises. An effective deterministic model is analytical derived where the noise parameters, intensity, correlation time, and correlation length appear explicitly. The different effects of these parameters are discussed for the Ginzburg-Landau and Schlögl models. We obtain an analytical expression for the front velocity as a function of the noise parameters. Numerical simulation results are in a good agreement with the theoretical predictions.
\end{abstract}

DOI: 10.1103/PhysRevE.64.016129

PACS number(s): 02.50.-r, 05.40.-a, 47.54.+r

\section{INTRODUCTION}

The role of external fluctuations in extended systems is a subject of very active research because of its relevance in pattern formation in nonequilibrium systems [1,2]. A simple example of a dynamical pattern is a front moving at constant velocity. Fronts can be easily modelized by a reactiondiffusion equation with two steady states of different stability [3]. The study of the front dynamics under the influence of noises is relevant not only from theoretical point of view [4-9] but also from practical point as recent works on chemical kinetics have shown [10-13]. In these experiments a chemical wave moves under the influence of an external fluctuating illumination that is projected in the reactive medium. This external source of noise has finite intensity, correlation time, and correlation length. Thus, in this system we deal with spatiotemporal structured noise and not with a white noise. The present work could be useful to clarify the role of these parameters on propagating structures as studied experimentally in [10].

Previous studies dealt with this problem under the simplified assumption of white ( $\delta$-correlated) external fluctuations [4-9]. Nevertheless one can ask about the correctness of this assumption to modelize real noises. To answer this question, at least it would be necessary to calculate the first contributions of the finite value external noise parameters. If these corrections are controlled then one can get confidence on the simplified assumption of white noise.

Since the early work of Schlögl et al. [14] on the effects of fluctuations on a chemical interface, an intensive work has been devoted to describe the related problem of front propagation in the presence of an external noise source [4-9]. A complete study for the case of a white noise was presented in $[8,9]$ in which the front velocity and its diffusive dispersion behavior was computed in terms of the effective white noise intensity. It was found that the velocity of the front increases with the noise intensity due to a systematic contribution to the kinetic terms. Actually the dispersion is subdiffusive for the so called pulled fronts, as has been shown recently [15]. A variety of approaches has been adopted, from projection

\footnotetext{
*Corresponding author. Email address: msantos@etse.urv.es
}

techniques [6,7] to scaling arguments [15], including a stochastic version of the multiple scale analysis as well as a nonsystematic noise expansion [9]. The last one may be the most simplified one that grasps the systematic contribution of the noise to the dynamics of the system. This contribution usually appears as a renormalization of the reaction parameters and it is the origin of the well known shift of the front velocity.

The general aim of this paper is to find what are the most relevant effects of a real noise on two different models that exhibit front propagation. We will see that for a fixed noise intensity, the noise correlation time is a relevant parameter that interpolates the results of the white noise limit with those of the deterministic case, but the role of the correlation length is different.

Here, we will derive an analytical expression of the effect of a spatiotemporal structured noise on an extended system governed by a Langevin reaction-diffusion equation with multiplicative noise. We will closely follow the guidelines settled in [16] for a one variable system, and also those in [17] for multivariable system to deal with nonwhite noises in extended systems. Our main difference is that our analysis is done in the continuum space, and also that we present a more simplified way to get the first order contribution of the noise in the correlation time $\tau$, which avoid the integration of a response function. Here we note that the continuum Langevin description may perfectly be adequate for describing reactive fronts even though chemical systems are discrete in nature, as was shown in [18].

Our theoretical predictions have been applied to two systems: the Ginzburg-Landau and the Schlögl models. In the first case the noise induces the front by controlling the stability of the new state versus the other unstable steady state. In the second case, the noise does not change the steady state but controls its dynamics.

The outline of the paper is as follows. Section II contains the main theoretical results and a discussion of some limiting cases. There we present the derivation of an effective dynamical equation that grasps the systematic contribution of the different noise parameters. In Sec. III, we apply these results to obtain explicit predictions for the two models already mentioned and we discuss the numerical results obtained for them and their comparison with the analytical pre- 
dictions. In Sec. IV we summarize our conclusions. Appendices are devoted to technical aspects of our analytical methodology and the implementation of a particular algorithm to generate a spatiotemporal structured noise.

\section{EFFECTIVE DYNAMICAL MODEL}

We consider the following stochastic partial differential equation as a representative description of reaction-diffusion systems under multiplicative fluctuations:

$$
\frac{\partial \psi(x, t)}{\partial t}=\mathcal{L}\left(\psi(x, t), \partial_{x}, a\right)+\epsilon^{1 / 2} g(\psi) \eta(x, t),
$$

where $\mathcal{L}$ is a reaction-diffusion operator that explicitly reads,

$$
\mathcal{L}\left(\psi(x, t), \partial_{x}, a\right)=D \frac{\partial^{2} \psi(x, t)}{\partial x^{2}}+f(\psi, a)
$$

$f(\psi, a)$ and $g(\psi)$ is the reaction term and the coupling term with external fluctuations, respectively, and $\eta(x, t)$ is a Gaussian spatiotemporal structured noise with the following statistical properties,

$$
\begin{gathered}
\langle\eta(x, t)\rangle=0 \\
\left\langle\eta\left(x^{\prime}, t^{\prime}\right) \eta(x, t)\right\rangle=G\left(\left|x-x^{\prime}\right|,\left|t-t^{\prime}\right|\right) \\
=C\left(\left|x-x^{\prime}\right|\right) \gamma\left(\left|t-t^{\prime}\right|\right) .
\end{gathered}
$$

Also, for simplicity but not strictly necessary, we have made the assumption that this correlation function factorizes in a spatial and temporal part. To fix the notation, and following the commonly accepted generic prescription [2], we define the three parameters of the noise, intensity, correlation time, and correlation length, as follows:

$$
\begin{gathered}
\sigma^{2} \equiv \int_{0}^{\infty} d s \int_{\mathcal{R}} d r G(r, s), \\
\tau \equiv \frac{1}{\sigma^{2}} \int_{0}^{\infty} d s \int_{\mathcal{R}} d r G(r, s) s, \\
\lambda^{2} \equiv \frac{1}{\sigma^{2}} \int_{0}^{\infty} d s \int_{\mathcal{R}} d r G(r, s) r^{2} .
\end{gathered}
$$

We pursue here to find the systematic and most relevant effects of this type of noise. In general, the noise has two important effects, systematic and fluctuating ones, which cannot be exclusively associated with the deterministic and stochastic terms of Eq. (1), respectively. In fact noise acts in two different scales [9]. Fast fluctuations in a short time scale modify the front shape and thus producing an effective front with different deterministic properties. On the other hand, the slow fluctuations are responsible for the diffusive dispersion of the front position.

A naive way to get these systematic effects of the fluctuations is by analyzing the noise term in Eq. (1). Due to the multiplicative character of the noise, although $\eta$ has zero mean, this is not the case for this stochastic term,

$$
\epsilon^{1 / 2}\langle g(\psi(x, t)) \eta(x, t)\rangle \equiv\langle\Phi(\{\psi\})\rangle \neq 0,
$$

which, as a consequence, will give a net contribution to the dynamics. This can be explicitly shown in the following way. By adding and substructing $\Phi(\{\psi\})$ to our original dynamical equation (1), we can write this equation as

$$
\begin{gathered}
\frac{\partial \psi(x, t)}{\partial t}=\mathcal{L}\left(\psi(x, t), \partial_{x}, a\right)+\Phi(\{\psi\})+\epsilon^{1 / 2} \mathcal{R}(\psi, x, t), \\
\epsilon^{1 / 2} \mathcal{R}(\psi, x, t) \equiv \epsilon^{1 / 2} g(\psi) \eta(x, t)-\Phi(\{\psi\}) .
\end{gathered}
$$

This dynamics is statistically equivalent to the original one. Note that for the new noise term it is $\langle\mathcal{R}(\psi, x, t)\rangle \equiv 0$ and it has a correlation which can be developed in powers of $\epsilon^{1 / 2}$. We make now the Ansatz that if the noise allows for a well definite front structure, its systematic behavior will be described by the deterministic equation

$$
\frac{\partial \psi(x, t)}{\partial t}=D \frac{\partial^{2} \psi(x, t)}{\partial x^{2}}+\Phi(\{\psi\})
$$

called the effective dynamics. In Appendices A and B we present a detailed calculation of $\Phi$ for small $\tau$, which is given by

$$
\Phi(\{\psi\})=\epsilon D C(0) \tau g^{\prime} g^{\prime \prime}\left(\frac{\partial \psi(x, t)}{\partial x}\right)^{2}+h(\psi(x, t)),
$$

where

$$
\begin{aligned}
h(\psi(x, t)) \equiv & f(\psi(x, t), a)+\epsilon\{C(0) \\
& \left.+D C^{\prime \prime}(0) \tau\right\} g(\psi(x, t)) g^{\prime}(\psi(x, t)) \\
& -\epsilon C(0) \tau g^{\prime}(\psi(x, t))\{g(\psi(x, t)), f(\psi(x, t))\}
\end{aligned}
$$

is the new effective reaction term. The brackets are defined as

$$
\{g, f\} \equiv g^{\prime} f-g f^{\prime},
$$

and the primes on $f(\psi)$ and $g(\psi)$ indicate the derivative with respect to $\psi$.

Thus, we have ended up with one of the most important results of this paper Eq. (7), which contains the systematic contribution of the noise to our original dynamics (1) up to first order in $\tau$. In this paper we will not study the effect of $\mathcal{R}$. This term is only relevant for those nonsystematic effects of the noise, such as, the dispersion of the front. The dependence on the parameter $\lambda$ is included in $C(0) \sim \sigma^{2} \lambda^{-1}$ and $C^{\prime \prime}(0) \sim \sigma^{2} \lambda^{-3}$. As will be seen below, $C(0)$ is the most relevant quantity. Thus the main effect of the correlation length through $C(0)$ is trivial. For this reason we will pay more attention to the nontrivial influence of the term $C^{\prime \prime}(0)$ fixing $C(0)$ independent of $\lambda$. 
A first check of the previous results will be provided by considering the better known case of temporal white noise in a lattice. Here we will first define the proper limit by which Eq. (3) becomes a temporal white noise, and then see if Eq. (7) correctly reproduces the results found in Ref. [8].

The temporal white noise in time has a correlation,

$$
\left\langle\eta\left(x^{\prime}, t^{\prime}\right) \eta(x, t)\right\rangle=2 C\left(x-x^{\prime}\right) \delta\left(t-t^{\prime}\right),
$$

where the spatial white noise limit is given by

$$
\lim _{\lambda \rightarrow 0} C\left(x-x^{\prime}\right)=\delta\left(x-x^{\prime}\right) .
$$

In a one-dimensional lattice this takes the form of

$$
\lim _{\lambda \rightarrow 0} C_{i j} \equiv \frac{\delta_{i j}}{\Delta x} .
$$

In this limit one can see that all the integrals in Eq. (A8) vanish except the first one. The systematic dynamics of Eq. (7) is then that of the effective reaction term given now by $\left(\sigma^{2}=1\right)$

$$
h_{\eta}(\psi(x, t)) \equiv f(\psi(x, t), a)+\epsilon(0) g(\psi(x, t)) g^{\prime}(\psi(x, t)),
$$

with $\epsilon(0) \equiv \epsilon C(0)=\epsilon / \Delta x$.

In this way we have recovered the results found in Ref. [8]. Note that one cannot consider right from the beginning a white noise in space because the ill defined $\delta(0)$.

The value of $C^{\prime \prime}(0)$ for a spatial white noise in the lattice is evaluated as

$$
C^{\prime \prime}(0)=\frac{C(1)-2 C(0)+C(-1)}{(\Delta x)^{2}}=-\frac{2}{(\Delta x)^{3}},
$$

where $C( \pm 1)=0$ has been used. For the case of a spatial structured noise with $\lambda$ finite, all the integrals in Eq. (A8) can be evaluated.

\section{APPLICATIONS AND NUMERICAL RESULTS}

We will now study the effects of a colored noise for two particular types of couplings $g(\phi)$ : a linear and a nonlinear one, which correspond to the Ginzburg-Landau and Schlögl models, respectively. The noise will enter in the standard way [19] as small fluctuations of the control parameter $a$,

$$
a \rightarrow a+\epsilon^{1 / 2} \eta(x, t),
$$

and thus the Langevin-type coupling function is given by

$$
g(\psi(x, t))=\frac{\partial f(\psi, a)}{\partial a} .
$$

Numerical simulations of Eq. (1) for the different models have been performed in a one-dimensional lattice of mesh size $\Delta x=0.5$. The length of the system $L=600$. We have used a Heun algorithm [2] with a time step $\Delta t=0.01$. In all cases, $D=1$ and $a=-0.1$, except for the Schlögl model, where different values of $a$ have been used.

The noise is generated with a spatial and temporal structure as a Gaussian random number at each lattice point. The correlation function factorizes as in Eq. (3). The temporal part has an exponential decay (Ornstein-Uhlenbeck process) with a correlation time $\tau$, while the spatial correlations have a triangular shape with a correlation length $\lambda$. The numerical implementation of such a noise is described in Appendix C.

The initial condition for the Ginzburg-Landau model is a small pulse with a height of 0.01 and located at the middle of the spatial domain. In this way the initial perturbation will spread off as two fronts propagate in opposite directions. For the Schlögl model the initial field is a steplike function of value $\psi(x, 0)=1$ within the left half, and $\psi(x, 0)=0$ in the rest of the spatial domain. The numerical calculation of the mean front velocity and the steady state behind the front have been done as in Ref. [20].

\section{A. Linear coupling: The Ginzburg-Landau model}

This model has already been considered in the context of noise-induced fronts [20]. We will now study how that picture is modified by a spatiotemporal structured noise. For this model the kinetic term is

$$
f(\psi, a)=-\psi\left(a+\psi^{2}\right),
$$

and as a consequence, the noise coupling term is linear,

$$
g(\phi)=\phi .
$$

We will have then that the effective dynamics given by Eq. (7) is

$$
\frac{\partial \psi(x, t)}{\partial t}=D \frac{\partial^{2} \psi(x, t)}{\partial x^{2}}+h(\psi(x, t))
$$

with a new kinetic term (9) given now as

$$
h(\psi(x, t))=-\psi\left(a^{\prime}+b^{\prime} \psi^{2}\right),
$$

where the new kinetic parameters are

$$
\begin{gathered}
a^{\prime}=a-\epsilon\left\{C(0)+D C^{\prime \prime}(0) \tau\right\} \\
b^{\prime}=1+2 \epsilon C(0) \tau .
\end{gathered}
$$

Following the linear marginal stability criteria [21], the velocity of a pulled front is controlled by the linear term as

$$
\begin{aligned}
v_{l}^{*} & =2 \sqrt{D\left(-a^{\prime}\right)} \\
& =2 \sqrt{D\left(-a+\epsilon(0)\left(1+\frac{D C^{\prime \prime}(0)}{C(0)} \tau\right)\right)} .
\end{aligned}
$$

Note that $a^{\prime}<0$ in order to have a front. This result, however, has been deduced for a small enough $\tau$. Nevertheless, we can conjecture a generalization of Eq. (23) for any value of $\tau$ considering that the values of the velocity for $\tau=0$ (temporal white noise limit) and for $\tau=\infty$ (deterministic 
case) are known. In this way, the simplest regularization of Eq. (23), which is a monotonous function on $\tau$, is

$$
v_{\tau}^{*}=2 \sqrt{D\left(-a+\frac{\epsilon(0)}{\left.1-\frac{D C^{\prime \prime}(0)}{C(0)} \tau\right)}\right.}
$$

Moreover, Eq. (24) generalizes the temporal white noise result in terms on a renormalized noise intensity defined as

$$
\epsilon_{\mathcal{R}} \equiv \frac{\epsilon(0)}{1-\frac{D C^{\prime \prime}(0)}{C(0)} \tau},
$$

which does not present any singularity because always $C^{\prime \prime}(0)<0$.

Taking $\epsilon(0)=\epsilon C(0)$ as a constant we have the following behavior. For increasing $\tau$ ( $\lambda$ fixed), $\epsilon_{\mathcal{R}}$ decreases, and we arrive up to the deterministic value of $v$. Nevertheless for fixed $\tau$ and increasing $\lambda, C^{\prime \prime}(0) \tau / C(0) \sim \tau / \lambda^{2}$, and then $\epsilon_{\mathcal{R}}$ increases, and as a consequence, $v$ increases up to the temporal white noise limit. This is a nontrivial effect of $\lambda$ that needs a finite value of $\tau$ to appear.

As already discussed in [20], another important quantity in this model is the field behind the front that is induced by the noise, and thus it is highly fluctuating. From Eq. (21), the homogeneous deterministic stationary value behind the front can be calculated as

$$
\psi_{s t}=\sqrt{\frac{-a^{\prime}}{b^{\prime}}} .
$$

As we do not know how the higher order corrections on $b^{\prime}$ are, we expect a poorer agreement for $\psi_{s t}$ than for the velocity. However, we can get an idea of the relevance of $b^{\prime}$ by numerically inspecting the quotient of $v_{\tau}^{*}$ over $\psi_{s t}$ that depends on $b^{\prime}$. Indeed, from Eqs. (23) and (26), this dependence is

$$
\left(b^{\prime}\right)^{1 / 2}=\frac{1}{2} \frac{v_{\tau}^{*}}{\psi_{s t} \sqrt{D}} .
$$

The analytical predictions (24), (26), and (27) are important results of this paper that will be checked numerically. Due to the different role of the noise parameters $\tau$ and $\lambda$ we will discuss two cases separately.

\section{Spatial white noise in the lattice}

For this case all the simulations agree perfectly with the theoretical results of Eq. (24). In fact, fixing the noise intensity $\epsilon(0)$ and increasing $\tau$, the mean velocity of the front drops monotonously to the deterministic value (see Fig. 1). All figures are in dimensionless units.

Our analytical calculation (dashed lines) only describes the corrections to the white noise case at order $O(\tau)$. However, this can be considered quite relevant as the dependence of $v$ and $\psi_{s t}$ on $\tau$ drops down very rapidly near $\tau=0$, and

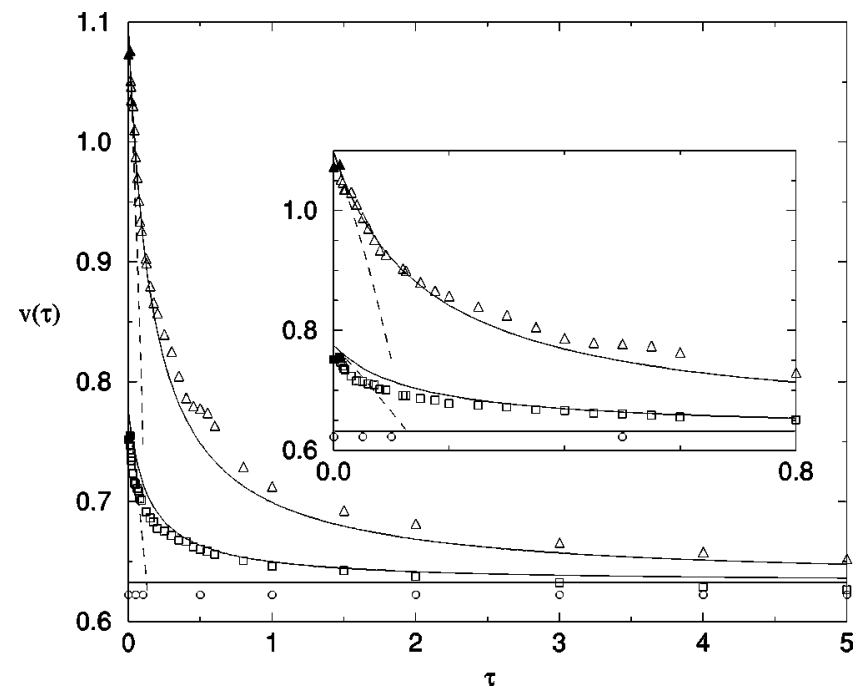

FIG. 1. Front mean velocity versus noise corelation time $\tau$ for the Ginzburg-Landau model in the presence of a OrnsteinUhlenbeck noise in time, white in space. Values of the parameters: Circles correspond to the deterministic case, $v_{\text {sim }}^{*}=0.62$; triangles, to $\epsilon(0)=0.2$, and squares, to $\epsilon(0)=0.05$; dashed lines are the analytical prediction up to $O(\tau)$ Eq. (23), whereas continuous lines represent the corrected prediction (24). See text for the values of the other parameters.

our first order approximation succeeds to grasp this pronounced slope (see inset of Fig. 1). Moreover, the extended analytical prediction (24) shows a very good agreement with numerical data for all values of $\tau$.

With respect to the average mean field, the agreement is more qualitative (see Fig. 2). The numerical results for the effective parameter $b^{\prime}$, evaluated from Eq. (27), are represented in Fig. 3. They support the initial growth of $b^{\prime}$ predicted by the theory. For greater values of the correlation time $b^{\prime}$ stays bounded by its deterministic value $b_{d}^{\prime}=1$. This fact may explain why numerical values of $\psi_{s t}$ seem to de-

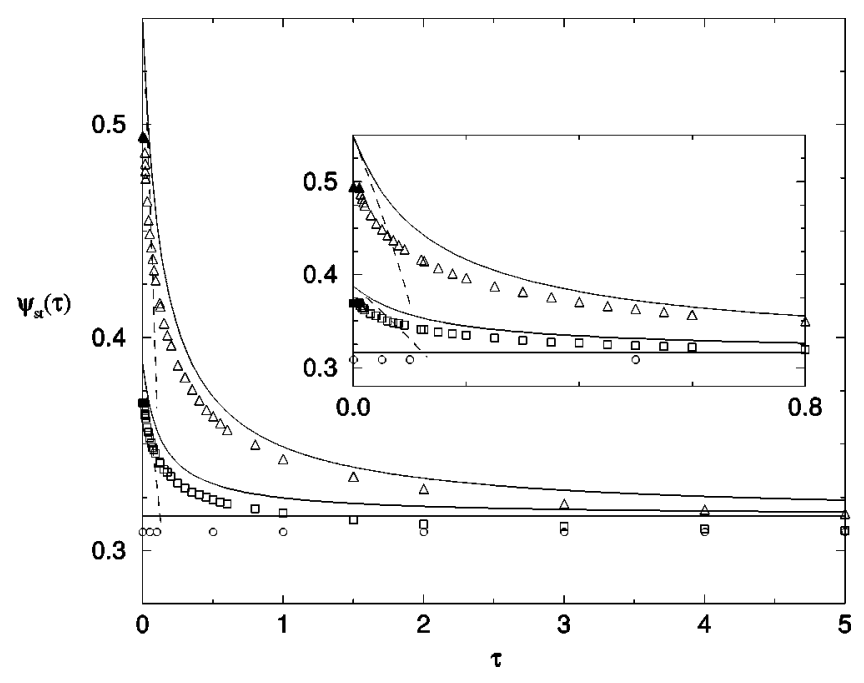

FIG. 2. Mean stationary value of the field versus $\tau$. The inset is a amplification of the small $\tau$ domain. See previous figure for the symbol notation. 


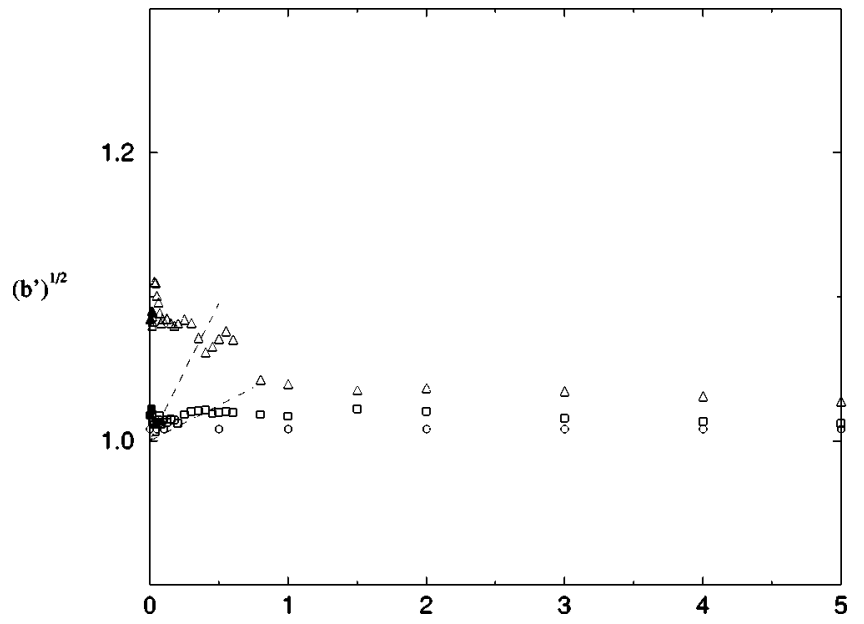

FIG. 3. Cubic term coefficient of the Ginzburg-Landau model as the quotient of $v$ to $\phi$ versus $\tau$. Notation is the same as in previous figures.

pend only on the linear coefficient $a^{\prime}$.

From previous work [20], we already know that $\psi_{s t}$ has a systematic error that slightly increases with noise intensity. Hence, the deviation found here (Fig. 3) is not due to the presence of temporal correlations, but a problem related to the fact that we are measuring a highly fluctuating quantity as it is $\psi_{s t}$. In any case, our theoretical prediction is consistent qualitatively with numerical simulation results.

\section{Spatiotemporal structured noise}

To study the nontrivial effects of a finite correlation length on the dynamics of the front, we have to pay attention to the effects coming from the quantity $C^{\prime \prime}(0) \sim \sigma^{2} / \lambda^{-3}$.

In Fig. 4 we can see the front velocity versus $\tau$ for different values of the correlation length $\lambda$ of the noise. Continuous lines correspond to the analytical prediction (24). As can be seen, for a finite correlation length the agreement is only qualitative, and improves for noises not too much away from

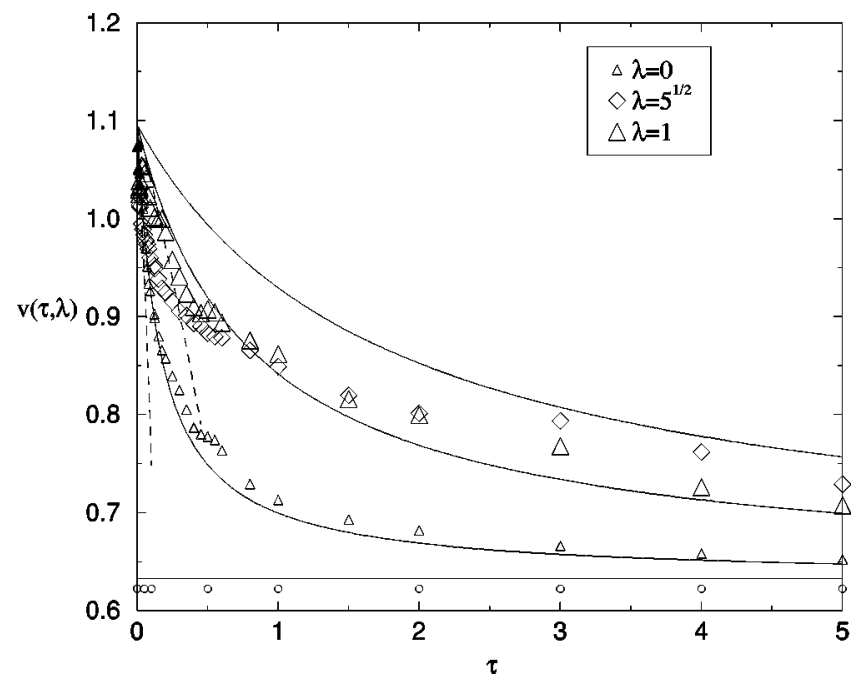

FIG. 4. Front mean velocity versus $\tau$ for different correlation lengths. Here it is $\epsilon(0)=0.2$.

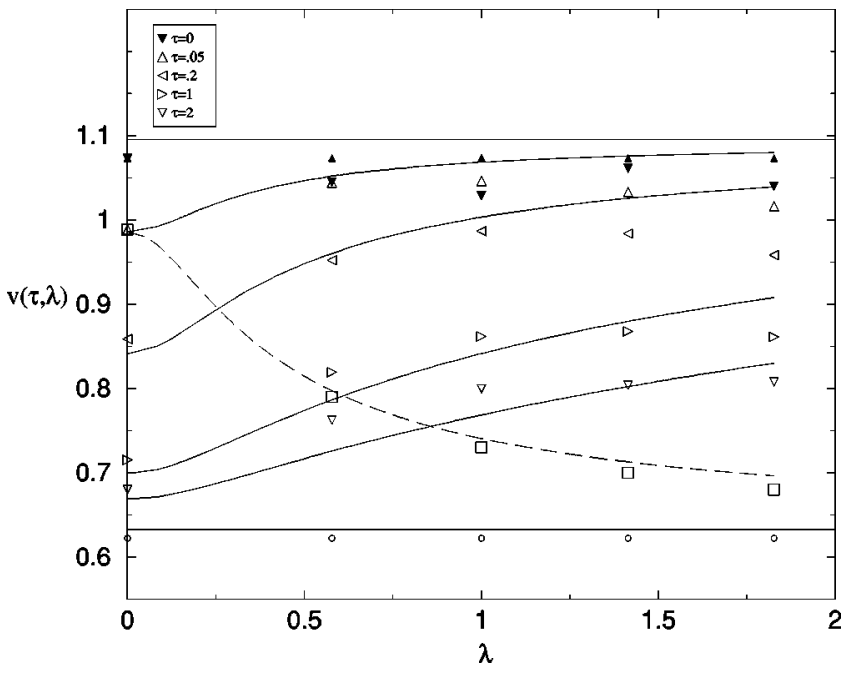

FIG. 5. Front mean velocity versus $\lambda$ for different correlation times. $\epsilon(0)=0.2$ for hollow triangles. Filled up-triangles correspond to the white noise case, while circles to the deterministic case. Squares correspond to the trivial noise effects with fixed noise intensity for $\tau=0.05$ and the long-dashed line is its theoretical prediction (24) (see text). Each set of vertical points along the horizontal axes correspond, in increasing order of $\lambda$, to $m=0,1,2,3,4$, respectively. See Appendix C.

a spatial white noise (triangles). This behavior can better be appreciated in Fig. 5 where we have plotted the velocity versus $\lambda$ for different values of the correlation time.

According to our definition of $\lambda$, Eq. (C8), we have that $\lambda=0$ for the spatial white noise in the lattice. Here it can be seen that our analytical scheme may qualitatively describe the effects of a finite $\lambda$ only for small values of $\tau$ and $\lambda$, which is not the case for $\lambda=0$ where the accordance is very good. For a finite $\lambda$, we have also observed a clear departure from the analytical results in the case of the dependence on $\tau$ of the mean stationary value of the field behind the front, as well as for the cubic coefficient. Also, the numerical results show a systematic decrease of the velocity when increasing the correlation length for $\tau=0$, which is not predicted by our analytical results (24).

On the other hand, for $\tau>0$, the velocity tends to grow with $\lambda$ for small correlation lengths as the theory predicts. Indeed, this agrees with what we have observed in preliminary numerical simulations for a quenched white noise. Hence, for increasing $\lambda$ ( $\tau$ fixed) the numerical results suggest a nonmonotonous behavior of the velocity, which may increase at small $\lambda$, but would always decrease at long correlation lengths.

We have not found yet an explanation for this effect. We believe that there is an interplay between the correlation length of the noise and the typical length of the front, which is given by its width. Indeed, this effect could be related to the observed distortion of the leading edge of the front and the possible formation of a prefront in the presence of a large spatial correlation length of the noise. Then our initial assumption of a well defined mean front profile is not fulfilled and, as a consequence, the theoretical scheme cannot be applied. 
For the sake of completeness, in this figure we also show what are the trivial effects of of a finite correlation length. In this case, the noise has been generated such that the noise intensity $\sigma^{2}$, defined in Eq. (4), remains constant. This can be acomplished by changing the previous weighting function $g_{i}$ by a factor $(\sqrt{2 m+1})^{-1}$ (see Appendix C). Squares correspond to the numerical results $(L=2400)$. The dashed line is the theoretical prediction (24) for this case. As can be seen, there is a monotonous decay of the velocity because now the most dominant term $\epsilon(0) \sim \sigma^{2} / \lambda$ also decays with $\lambda$. Thus, the qualitative behavior is completely different. This confirms that the previous studied dependence on $\lambda$ correspond indeed to a nontrivial effect of the correlation length. Note that our theoretical scheme succeeds better in describing quantitatively the trivial effects of a finite $\lambda$.

\section{B. Nonlinear coupling: The Schlögl model}

A general model was introduced by Schlögl in [14] when studying the fluctuations of an interface. Here we will consider a particular version of it that was studied in the presence of an external white noise in Ref. [8]. It corresponds to the reaction term

$$
f(\psi, a)=-\psi(\psi+a)(\psi-1),
$$

which implies a nonlinear coupling with the noise,

$$
g(\psi(x, t))=\psi(\psi-1) .
$$

Taking into account these definitions, the effective deterministic part of Eq. (7) becomes,

$$
\begin{aligned}
\frac{\partial \psi(x, t)}{\partial t}= & D \frac{\partial^{2} \psi(x, t)}{\partial x^{2}}+h(\psi(x, t))+D \epsilon(0) \tau 2(2 \psi-1) \\
& \times\left(\frac{\partial \psi(x, t)}{\partial x}\right)^{2}
\end{aligned}
$$

where the reaction term is

$$
h(\psi(x, t))=a^{\prime} \psi+b^{\prime} \psi^{2}+c^{\prime} \psi^{3}+5 d^{\prime} \psi^{4}-2 d^{\prime} \psi^{5},
$$

with the effective kinetic parameters,

$$
\begin{gathered}
a^{\prime}=a+\epsilon_{\mathcal{R}}, \\
b^{\prime}=1-a+d^{\prime}-3 \epsilon_{\mathcal{R}}, \\
c^{\prime}=-1-4 d^{\prime}+2 \epsilon_{\mathcal{R}}, \\
d^{\prime}=\epsilon(0) \tau,
\end{gathered}
$$

where $\epsilon_{\mathcal{R}}$ was defined in Eq. (25).

Our point of interest in this model is the mean front velocity because the steady states for the front $\psi=0,1$ are not modified by the noise. Due to the prefactor of the KPZ-like term, for $\psi>1 / 2$, any deviation from the homogeneous state $\psi=1$ tends to grow, while this is opposite at points where $\psi<1 / 2$ for any deviation from the state $\psi=0$. Thus, the effect of this term is to shorten the width of the front, i.e., to select a greater decay mode of the front, thus slowing down its propagation [21]. Hence, the expected slowing down of the front due to temporal correlations of the noise arises here in two ways: The first one is by means of the usual renormalization of the coefficients of the reaction term $f(\psi)$. The second type of corrections come from the new Kadar-ParisiZhang (KPZ)-like term.

We expect that this front will exhibit the general regimes of front propagation [21], i.e., a linear (pulled front), a nonlinear and a metastable (both, pushed front) regimes. In the linear regime, the velocity depends only on the linear coefficient of the reaction term $a^{\prime}$, and it is then given by Eq. (24).

While a crossover from a metastable to a nonlinear regime is trivial to determine, being nothing more than condition $a^{\prime}=0$, the transition between the linear and the nonlinear regimes is far more complicated to locate. This calculation of this point requires an analysis of (all) higher power terms of the reaction, determining a complete solution in the comoving system and requiring then that the asymptotic behavior is such that the coefficient of the slowest decay mode vanishes.

For the case of a temporal white noise, as was found in $[8,9]$, there is only a renormalization of the parameters of the kinetic terms, in such a way that the effective dynamics is equivalent to the deterministic one up to a rescaling of the coefficients. Hence, the location of the different regimes can be directly determined from those of the deterministic case. Unfortunately, to our knowledge, the first procedure is hopeless for Eq. (30). Neither can this dynamic directly be compared with the deterministic case. However, the transition between linear and nonlinear regime is always continuous and, as we can correctly describe the linear regime, this fact will help us to numerically locate the transition for this model.

Nevertheless, there is still some hope for an analytical prediction. The type of dynamics given by Eqs. (30)-(32) usually are relevant near the transition point $a^{\prime}=0$, where the dynamics given by Eq. (2) can be simplified by means of an amplitude expansion. In this case, and as long as the noise intensity is low enough, our effective equation would also lie near threshold $\left(a^{\prime} \sim 0\right)$. Assuming this situation, the spatial variations of the field take place on a typical length scale of order $q_{o}^{-1} \equiv \sqrt{D / a}$. A crossover between nonlinear and linear regime means that the nonlinear terms start to dominate the growth rate of the initial steady state. Thus, this transition takes place when both $a^{\prime} \psi$ and $b^{\prime} \psi^{2}$ are of the same order of magnitude. This will be the case for

$$
\psi \sim b^{\prime} ; \quad b^{\prime 2} \sim a^{\prime}
$$

Then the KPZ and the $\psi^{4}$ terms both will be of order $q_{o}^{4}$, while the term $\psi(\partial \psi(x, t) / \partial x)^{2}$ will be of order $q_{o}^{5}$. Hence, near threshold, only the first three terms of the effective reaction (31) will be relevant. But this equation is just the standard Schlögl model that is exactly solvable. This will have a sense only if it is $c^{\prime}<0$, which we will assume to be 


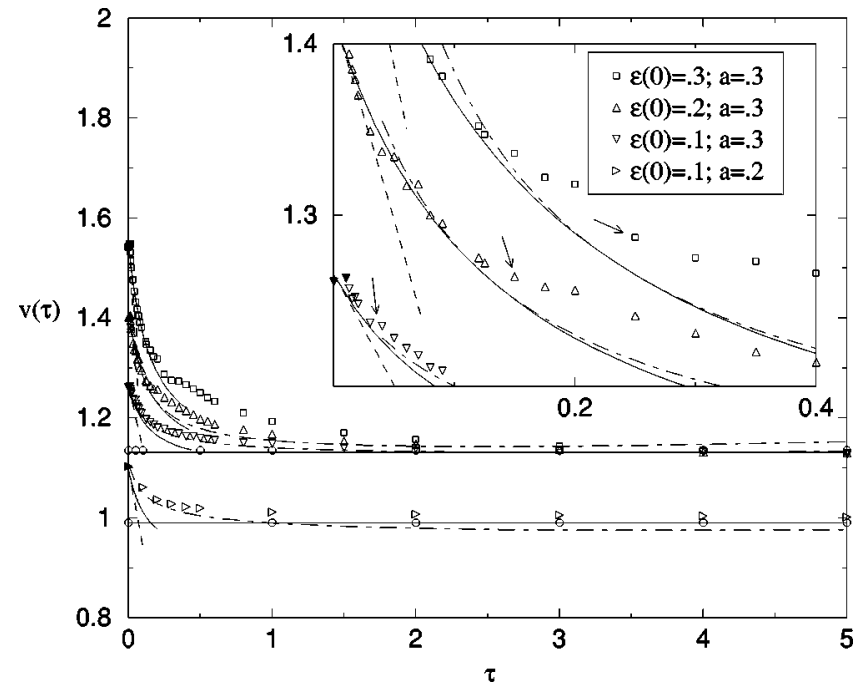

FIG. 6. Front mean velocity versus noise corelation time $\tau$ for the Schlögl model. Circles correspond to the deterministic case, $v_{d, \text { sim }}^{*}=1.131$; dashed lines are the analytical results up to $O(\tau)$, whereas continuous lines represent the analytical corrected results $v_{\tau}^{*}$ Eq. (24). Dot-dashed lines correspond to the prediction (36). The inset amplifies the domain of small $\tau$. By tuning $\tau$, the front shifts from a pulled regime to a pushed one. This crossover corresponds to the points first leaving the theoretical curve $v_{\tau}^{*}$ and are approximately given by the arrows.

so due to its expression in Eq. (32). As stated above, we will also require $b^{\prime}>0$. The new stationary states of this approximation are given by

$$
\psi_{ \pm} \equiv\left(\frac{-b^{\prime}}{2 c^{\prime}}\right)\left\{1 \pm \sqrt{1-\frac{4 a^{\prime} c^{\prime}}{b^{\prime 2}}}\right\}
$$

For $a^{\prime} c^{\prime}<0$ and $b^{\prime}>0$, it is $\psi_{+}>\psi_{-}$. If we write then the reaction term as

$$
h_{c r}(\psi(x, t))=c^{\prime} \psi\left(\psi-\psi_{+}\right)\left(\psi-\psi_{-}\right),
$$

the velocity of a front connecting $\psi=\psi_{+}$and $\psi=0$ is given by [22]

$$
v_{n l}=\sqrt{-2 c^{\prime} D}\left\{\frac{\psi_{+}}{2}-\psi_{-}\right\} .
$$

Given the results found for the previous model, we expect that our system (31) will present fronts in the linear regime for a high enough noise intensity $\epsilon(0)$ and small enough $\tau$.

Although we do not know the corrections of $d^{\prime}$ beyond $O(\tau)$, we expect the velocity to start diverging from Eq. (24) for some finite value of $\tau$. But, by adequately choosing the parameters, the transition between the linear and nonlinear regimes can be obtained at small enough values of $\tau$ for our approximations to be applicable. Hence, for values of the $a^{\prime}$ near threshold, and within a neighborhood of the crossing point, the nonlinear velocity $v_{n l}$ will approximately be given by Eq. (36).

We will show that numerical simulations support this analysis. In Fig. 6 we have plotted the numerical results of the front mean velocity versus $\tau$. The inset shows the cases where a linear-nonlinear transition is expected. For all three plots, the value of the deterministic linear coefficient $a$ is such that the deterministic front lies right within the nonlinear regime. While for the white noise case all fronts move within the linear regime, the one for $\epsilon(0)=0.1$ is only marginally inside. Increasing then $\tau$, the effective linear coefficient $a^{\prime}$ decreases. For small values of the correlation time, the fronts will still lie within the linear regime, except for the $\epsilon(0)=0.1$ case, for which the front enters the nonlinear regime immediately for any finite value of $\tau$.

Thus for small values of $\tau$ the front moves with the linear velocity. We can see that our first order approximation (dashed lines) also reproduces for this system the initial steep fall of $v$. Our analytical continuation $v_{\tau}^{*}$ shows up a perfect agreement with the numerical results. By further increasing the correlation time we can shift the front into the nonlinear regime.

For this one we only have a rough approximation for the front mean velocity given by Eq. (36) (dot-dashed lines). This approximation is valid only near the critical point $a^{\prime}$ $=0$ and gets worse as we move away from it. Although our analysis gives us only the corrections due to a finite $\tau$ up to $O(\tau)$, with the renormalized noise intensity (25), and staying close to the critical point, we obtain a surprisingly good analytical estimation for the nonlinear velocity up to values of $\tau$ of order $O(1)$. One can notice that our predictions are just $O(\tau)$ seeing that the velocity diverges from the deterministic value at $\tau$ high enough. This is to be expected as in obtaining Eq. (36) we have used only an $O(\tau)$ approximation for the squared $b^{\prime} \psi^{2}$ and the stabilizing term $c^{\prime} \psi^{3}$, with both $b^{\prime}$ and $c^{\prime}$ linearly increasing with $\tau$ for high values of $\tau$. But this dependence is obviously incorrect as an infinite energy difference between two metastable states would give rise to an unbounded propagation velocity, which is not the case.

The estimation of the crossing point between the linear and the nonlinear regimes, given by the crossing point between the two curves $v_{n l}^{*}$ and $v_{\tau}^{*}$, is consistent with the numerical results. At that point, these show up a pronounced and increasing departure of the linear velocity $v_{\tau}^{*}$. For the $\epsilon(0)=0.1$ case, the departure from the theoretical result $v_{\tau}^{*}$ starts already for $\tau>0$. This can be best seen in the inset of Fig. 6.

\section{CONCLUSIONS}

We have studied a general reaction-diffusion system that exhibits fronts in the presence of spatiotemporal structured external noise. We have derived an effective deterministic dynamical equation for the front that contains the main effects of the noise. These show up as renormalization of the original reaction terms of the deterministic system and also new terms. The relevance of those effects are discussed for two prototype models: The Ginzburg-Landau model (noise linear coupling) and the Schlögl model (noise nonlinear coupling). Although our analysis is valid only for small correlation times, we are able to grasp at least the main features of the effects of a time colored noise on an extended system, namely, that there is a pronounced slow down of the front 
velocity already for small but finite correlation times $\tau$.

We have obtained an analytical expression for the front velocity in the linear regime that we have extended to be valid for any value of the correlation time $\tau$ of the noise. The numerical simulations present an excellent agreement with the analytical results in this regime. For the nonlinear regime, we can only give an approximate expression for the velocity, when the system is near threshold. Although it still is valid only for $\tau$ not too large, it goes beyond the first order approximation considered in our analysis when the system is brought close enough to threshold.

Finally, we have obtained the nontrivial influence of the noise correlation length. Our results here are of a more limited validity, and this case needs a further study. Nevertheless, our numerical results suggest a nontrivial behavior of the velocity when varying the correlation length of the noise, with an increase of the velocity with $\lambda$ for finite $\tau$ and small $\lambda$. Previous reported studies evaluate only the trivial dependence on $\lambda$ of the front velocity, slowing down with increasing correlation length [23].

Hence, we have shown that our procedure of separating the systematic contribution of the noise from the original dynamics gives reliable information for front dynamics in the presence of spatiotemporal structured noises. The systematization of the present procedure, if possible at all, and its application to other situations would be extremely interesting.

\section{ACKNOWLEDGMENTS}

We acknowledge financial support from the Ministerio de Ciencia y Tecnología (Spain) under Project No. BFM20000624. M.A.S acknowledges financial support from the Departament d'Universitats, Recerca i Societat de la Informació, Generalitat de Catalunya. M.A.S is also very pleased to thank Professor L. Schimansky-Geier for fruitful comments and his hospitality during a stay at the Institut für Physik, Humbolt Universität zu Berlin, where part of this work was done.

\section{APPENDIX A: ANALYTICAL DERIVATION OF THE SYSTEMATIC EFFECTS OF A STRUCTURED NOISE}

The systematic contribution $\langle\Phi(x, t)\rangle$ of the noise is given by

$$
\langle\Phi(x, t)\rangle \equiv \epsilon^{1 / 2}\langle g(\psi(x, t)) \eta(x, t)\rangle .
$$

This average can be calculated by using Novikov's theorem in the following form,

$$
\begin{aligned}
\langle\Phi(x, t)\rangle= & \epsilon^{1 / 2} \int_{\mathfrak{R}} d x^{\prime} \int_{0}^{t} d t^{\prime} G\left(\left|x-x^{\prime}\right|,\left|t^{\prime}-t\right|\right) \\
& \times\left\langle g^{\prime}(\psi(x, t)) \frac{\delta \psi(x, t)}{\delta \eta\left(x^{\prime}, t^{\prime}\right)}\right\rangle .
\end{aligned}
$$

Hence, the determination of $\langle\Phi(x, t)\rangle$ reduces to that of the response function

$$
Q\left(x, x^{\prime} ; t, t^{\prime}\right) \equiv \frac{\delta \psi(x, t)}{\delta \eta\left(x^{\prime}, t^{\prime}\right)} .
$$

Following [16] and [17], we will consider the contribution of the noise at first order in $\tau$ in the approximation of small $\tau$. This means that temporal correlation decays very strongly for $t^{\prime} \neq t$ leaving relevant in Eq. (B3) only the values of the integrant for $t^{\prime}$ close to $t$. Thus we may expand $Q\left(x, x^{\prime} ; t, t^{\prime}\right)$ in powers of $\left(t^{\prime}-t\right)$ around $t^{\prime}=t$ and take all up to the first order

$$
Q\left(x, x^{\prime} ; t, t^{\prime}\right)=Q\left(x, x^{\prime} ; t, t\right)+\left.\frac{\partial Q}{\partial t^{\prime}}\right|_{t^{\prime}=t}\left(t^{\prime}-t\right)+\cdots
$$

In Appendix B we present a detailed derivation of this second term.

Now we can rearrange Eq. (A2) in two terms, the first one being the zero order or white noise contribution, i.e, the one we get in the limit $\tau \rightarrow 0$ for fixed $\epsilon$ and $\lambda$, while the second one represents the contribution of the colored noise at first order in $\tau$,

$$
\langle\Phi(x, t)\rangle=\left\langle\Phi_{0}(x, t)\right\rangle+\left\langle\Phi_{1}(x, t)\right\rangle .
$$

Recollecting relations (A2), (A4) and (B4), (B7), and after calculating the spatial integral, we obtain

$$
\begin{aligned}
\left\langle\Phi_{0}(x, t)\right\rangle= & \epsilon\left\langle g(\psi(x, t)) g^{\prime}(\psi(x, t))\right\rangle \int_{0}^{t} d t^{\prime} G\left(0,\left|t-t^{\prime}\right|\right) \\
\left\langle\Phi_{1}(x, t)\right\rangle= & \epsilon\left\langle g^{\prime}(\psi(x, t))[[\{g(\psi(x, t)), f(\psi(x, t))\}\right. \\
& \left.-D g^{\prime \prime}(\psi(x, t))\left(\frac{\partial \psi(x, t)}{\partial x}\right)^{2}\right] \int_{0}^{t} d t^{\prime} G\left(0,\left|t-t^{\prime}\right|\right) \\
& \times\left(t^{\prime}-t\right)-D g(\psi(x, t)) \int_{0}^{t} d t^{\prime} G^{\prime \prime}\left(0,\left|t-t^{\prime}\right|\right) \\
& \left.\left.\times\left(t^{\prime}-t\right)\right]\right\rangle,
\end{aligned}
$$

where the primes on $G\left(0,\left(t-t^{\prime}\right)\right)$ indicate derivatives of $G\left(\left(x-x^{\prime}\right),\left(t-t^{\prime}\right)\right)$ with respect to $x^{\prime}$, evaluated at $x^{\prime}=x$, and

$$
\begin{aligned}
\{g(\psi(x, t)), f(\psi(x, t))\} \equiv & g^{\prime}(\psi(x, t)) f(\psi(x, t), a) \\
& -g(\psi(x, t)) f^{\prime}(\psi(x, t), a) .
\end{aligned}
$$

As we are interested in the approximation of small $\tau$, which amounts to consider observation times much greater than the characteristic correlation time of the noise, we can extend then the limits of these integrals up to $\infty$.

At this point, further assumptions on the correlation function must be done in order to obtain any analytical prediction. Assuming that $G(x, s)$ factorizes as in Eq. (3), the above integrals can be written as 


$$
\begin{gathered}
\int_{0}^{t} d t^{\prime} G\left(0,\left|t-t^{\prime}\right|\right)=C(0) \sim \frac{\sigma^{2}}{\lambda} \\
\int_{0}^{t} d t^{\prime} G\left(0,\left|t-t^{\prime}\right|\right)\left(t^{\prime}-t\right)=-\tau C(0) \sim \frac{\sigma^{2} \tau}{\lambda} \\
\int_{0}^{t} d t^{\prime} G^{\prime \prime}\left(0,\left|t-t^{\prime}\right|\right)\left(t^{\prime}-t\right)=-\tau C^{\prime \prime}(0) \sim \frac{\sigma^{2} \tau}{\lambda^{3}},
\end{gathered}
$$

where the temporal part $\gamma(s)$ is considered to be normalized to 1 .

Finally, we can write

$$
\begin{aligned}
\left\langle\Phi_{0}(x, t)\right\rangle=\epsilon C(0)\left\langle g(\psi(x, t)) g^{\prime}(\psi(x, t))\right\rangle, & \text { (A9) } \\
\left\langle\Phi_{1}(x, t)\right\rangle= & -\epsilon C(0) \tau\left\langle g^{\prime}(\psi(x, t))[\{g(\psi(x, t)), f(\psi(x, t))\}\right. \\
& \left.\left.-D g^{\prime \prime}(\psi(x, t))\left(\frac{\partial \psi(x, t)}{\partial x}\right)^{2}\right]\right\rangle \\
& +\epsilon D C^{\prime \prime}(0) \tau\left\langle g^{\prime}(\psi(x, t)) g(\psi(x, t))\right\rangle .
\end{aligned}
$$

\section{APPENDIX B: RESPONSE FUNCTION}

The determination of $\langle\Phi(x, t)\rangle$ reduces to that of the response function

$$
Q\left(x, x^{\prime} ; t, t^{\prime}\right) \equiv \frac{\delta \psi(x, t)}{\delta \eta\left(x^{\prime}, t^{\prime}\right)}
$$

The meaning of Eq. (1) is given by

$$
\begin{aligned}
\psi(x, t+\Delta t)-\psi(x, t)= & \int_{t}^{t+\Delta t} d s \mathcal{L}\left(\psi(x, s), \partial_{x}, a\right) \\
& +\epsilon^{1 / 2} \int_{t}^{t+\Delta t} d s g(\psi(x, s)) \eta(x, s),
\end{aligned}
$$

and as long as $\tau>0$, the last integral is well defined as a Riemann integral. By taking the functional derivate of Eq. (B2) with respect to the noise $\eta\left(x^{\prime}, t^{\prime}\right)$ we get

$$
\begin{aligned}
& Q\left(x, x^{\prime} ; t, t^{\prime}\right)= Q\left(x, x^{\prime} ; t^{\prime}, t^{\prime}\right)+\int_{t^{\prime}}^{t} d s\left\{\frac{\partial \mathcal{L}}{\partial \psi}(x, s)\right. \\
&\left.+\epsilon^{1 / 2} g^{\prime}(\psi(x, s)) \eta(x, s)\right\} Q\left(x, x^{\prime} ; s, t^{\prime}\right) \\
& t>t^{\prime}
\end{aligned}
$$

with

$$
Q\left(x, x^{\prime} ; t, t\right)=\epsilon^{1 / 2} g(\psi(x, t)) \delta\left(x^{\prime}-x\right) .
$$

Equation (B3) is an integrodifferential equation for the response function for which it has not yet been found a formal solution as it was in [16] and [17] for nonspatially dependent (zero-dimensional) systems. The term (B4) gives the contri- bution (A9) to the systematic effect of the noise. Expanding $Q\left(x, x^{\prime} ; t, t^{\prime}\right)$ in powers of $\left(t^{\prime}-t\right)$ around $t^{\prime}=t$ and taking all up to the first order

$$
Q\left(x, x^{\prime} ; t, t^{\prime}\right)=Q\left(x, x^{\prime} ; t, t\right)+\left.\frac{\partial Q}{\partial t^{\prime}}\right|_{t^{\prime}=t}\left(t^{\prime}-t\right)+\cdots .
$$

The second term can be obtained by directly deriving Eq. (B3) with respect to $t^{\prime}$. This gives

$$
\frac{\partial Q\left(x, x^{\prime} ; t, t^{\prime}\right)}{\partial t^{\prime}}=\int_{t^{\prime}}^{t} d s \mathcal{I}(x, s)-\left\{\frac{\partial \psi\left(x, t^{\prime}\right)}{\partial t^{\prime}}, Q\left(x, x^{\prime} ; t, t^{\prime}\right)\right\} \text {. }
$$

As long as we are interested in the limit $t^{\prime} \rightarrow t$, the details of $\mathcal{I}(x, s)$ are not important for the first term in Eq. (B6) vanishes in that limit since it is a regular function in $s$. For $t^{\prime}$ $=t$, and substituting Eq. (1),

$$
\begin{aligned}
\left.\frac{\partial Q\left(x, x^{\prime} ; t, t^{\prime}\right)}{\partial t^{\prime}}\right|_{t^{\prime}=t}= & \left.\frac{\partial Q\left(x, x^{\prime} ; t^{\prime}, t^{\prime}\right)}{\partial \psi\left(x, t^{\prime}\right)}\right|_{t^{\prime}=t}\left(\mathcal{L}\left(\psi(x, t), \partial_{x}, a\right)\right. \\
& \left.+\epsilon^{1 / 2} g(\psi(x, t)) \eta(x, t)\right)-\left(\frac{\partial \mathcal{L}}{\partial \psi}(x, t)\right. \\
& \left.+\epsilon^{1 / 2} g^{\prime}(\psi(x, t)) \eta(x, t)\right) Q\left(x, x^{\prime} ; t, t\right) .
\end{aligned}
$$

Considering the initial condition (B4) and substituting the expression of the nonlinear (differential) operator $\mathcal{L}$ given by Eq. (1), the last relation reduces to

$$
\begin{gathered}
\left.\frac{\partial Q\left(x, x^{\prime} ; t, t^{\prime}\right)}{\partial t^{\prime}}\right|_{t^{\prime}=t} \\
=\epsilon^{1 / 2}\left[g^{\prime}(\psi(x, t)) f(\psi(x, t), a)-g(\psi(x, t)) f^{\prime}(\psi(x, t), a)\right] \\
\quad \times \delta\left(x^{\prime}-x\right)+D \epsilon^{1 / 2}\left\{g^{\prime}(\psi(x, t))\left(\frac{\partial^{2} \psi(x, t)}{\partial x^{2}}\right) \delta\left(x^{\prime}-x\right)\right. \\
\left.-\frac{\partial^{2}}{\partial x^{2}}\left[g(\psi(x, t)) \delta\left(x^{\prime}-x\right)\right]\right\} .
\end{gathered}
$$

The second order derivative of the last term gives

$$
\begin{aligned}
& {\left[g^{\prime \prime}(\psi(x, t))\left(\frac{\partial \psi(x, t)}{\partial x}\right)^{2}+g^{\prime}(\psi(x, t))\left(\frac{\partial^{2} \psi(x, t)}{\partial x^{2}}\right)\right] \delta\left(x^{\prime}-x\right)} \\
& \quad+2 g^{\prime}(\psi(x, t))\left(\frac{\partial \psi(x, t)}{\partial x}\right) \frac{\partial \delta\left(x^{\prime}-x\right)}{\partial x} \\
& \quad+g(\psi(x, t)) \frac{\partial^{2} \delta\left(x^{\prime}-x\right)}{\partial x^{2}}
\end{aligned}
$$

With this result in mind, the Laplacian terms in Eq. (B8) mutually cancel, while the terms proportional to the $\delta$ give 
rise to the first integral in Eq. (A6). Taken into account the following relation of the derivative of a $\delta$

$$
\frac{\partial}{\partial x} \delta\left(x^{\prime}-x\right)=-\frac{\partial}{\partial x^{\prime}} \delta\left(x^{\prime}-x\right)
$$

the contribution to Eq. (A2) of the first and second order derivative of the $\delta$ in (B9) will be

$$
\begin{aligned}
& -\int_{0}^{t} d t^{\prime}\left[2 G^{\prime}\left(0,\left(t-t^{\prime}\right)\right) g^{\prime}(\psi(x, t)) \frac{\partial \psi(x, t)}{\partial x}\right. \\
& \left.+G^{\prime \prime}\left(0,\left(t-t^{\prime}\right)\right) g(\psi(x, t))\right]\left(t^{\prime}-t\right) .
\end{aligned}
$$

This result give rise to the last terms in Eq. (A10), where the contribution proportional to $G^{\prime}\left(0,\left(t-t^{\prime}\right)\right)$ has been discarded because of the spatial isotropy of the noise.

\section{APPENDIX C: NUMERICAL ALGORITHM FOR GENERATING A SPATIOTEMPORAL COLORED NOISE}

Here, we will define a spatiotemporal structured noise that is very simple to implement numerically, and which is the one we have used in this work. This type of noise is obtained by rewriting the spectral method [2] as a linear transformation of a more simple noise field in real space.

We define our spatiotemporal colored noise in each lattice cell $i$ and at time $t$, as

$$
\eta_{i}(t) \equiv \Delta x \sum_{i} \bar{\eta}_{j}(t) g_{i-j}
$$

where the index $j$ labels a domain of cells around the cell $i$, and $g_{i}$ is a weighting distribution with the isotropic property

$$
g_{-i}=g_{i} \text {. }
$$

$\bar{\eta}_{i}(t)$ is an Ornstein-Uhlenbeck process in the lattice cell $i$, statistically independent of the other lattice points (white noise in space). Its value is generated through the linear Langevin equation,

$$
\frac{\partial \bar{\eta}_{i}(t)}{\partial t}=-\frac{1}{\tau} \bar{\eta}_{i}(t)+\frac{1}{\tau} \xi_{i}(t)
$$

in terms of a Gaussian white noise with a correlation,

$$
\left\langle\xi_{i}\left(t^{\prime}\right) \xi_{j}(t)\right\rangle=2 \sigma_{w}^{2} \frac{\delta_{i, j}}{\Delta x} \delta\left(t-t^{\prime}\right) .
$$

In this case the correlation of the noise $\bar{\eta}_{i}(t)$, is given by

$$
\begin{aligned}
\left\langle\bar{\eta}_{l+j}(s) \bar{\eta}_{j}(0)\right\rangle & =\bar{G}_{l}(s)=\bar{C}_{l} \gamma(|s|), \\
\gamma(|s|) & =\frac{\sigma_{w}^{2}}{\tau} e^{-|s| / \tau} \\
\bar{C}_{l} & =\frac{\delta_{0, l}}{\Delta x}
\end{aligned}
$$

Being $\bar{G}$ already factorized, the linear transformation (C1) assures that the correlation function of $\eta_{i}(t)$ will be of the desired form (3). As $g$ is arbitrary, we are free to impose the condition that the value of $G_{0}(0)$ equals that of $\bar{G}_{0}(0)$, i.e,

$$
G_{0}(0)=\bar{G}_{0}(0) \text {. }
$$

Here we are interested in $g_{l}$ having a finite range, for simplicity, we assume that $g_{i}$ is a constant $g$ inside the interval $-m<i<m$, but zero otherwise. Then the condition (C6) implies $g_{i}=[\Delta x \sqrt{(2 m+1)}]^{-1}$. Now it is a simple calculation to show that $\eta_{i}(t)$ is a spatiotemporal structured noise with a correlation,

$$
G_{l}=C_{l} \gamma(|s|)=\frac{\bar{C}_{0}}{(2 m+1)}[2 m+1-|l|] \theta(2 m-|l|) \gamma(|s|) .
$$

At equal lattice points this function decays exponentially in time, and at equal times it has a triangular decay as a function of lattice point difference. From this analytical expression it is straightforward to obtain the noise intensity and correlation length,

$$
\begin{gathered}
\sigma^{2}=(2 m+1) \sigma_{w}^{2} \\
\lambda=\sqrt{\frac{2}{3} m(m+1) \Delta x}
\end{gathered}
$$

being the correlation time $\tau$. One can check now that in the lattice white noise limit $m=0$ and $\sigma^{2}=\sigma_{w}^{2}$, and then $\lambda=0$. For $m=1$, we get that $\lambda=1.15 \ldots \Delta x$.
[1] A.S. Mikhailov, Phys. Rep. 184, 307 (1989).

[2] J. García-Ojalvo and J.M. Sancho, Noise in Spatially Extended Systems (Springer-Verlag, New York, 1999).

[3] M.C. Cross and P.C. Hohenberg, Rev. Mod. Phys. 65, 851 (1993).

[4] A.S. Mikhailov et al., Phys. Lett. 96A, 453 (1983).

[5] A. Engel, Phys. Lett. 113A, 139 (1985).

[6] L. Schimansky-Geier and Ch. Zúlicke, Z. Phys. B: Condens. Matter 82, 157 (1991).

[7] F. de Pasquale et al., J. Phys. A 25, 433 (1992).

[8] J. Armero et al., Phys. Rev. Lett. 76, 3045 (1996).
[9] J. Armero et al., Phys. Rev. E 58, 5494 (1998).

[10] I. Sendiña-Nadal, A.P. Munuzuri, D. Vives, V. PérezMuñuzuri, J. Casademunt, L. Ramírez-Piscina, J.M. Sancho, and F. Sagués, Phys. Rev. Lett. 80, 5437 (1998).

[11] S. Kàdàr, J. Wang, and K. Showalter, Nature (London) 391, 770 (1998).

[12] J. Wang, S. Kàdàr, P. Jung, and K. Showalter, Phys. Rev. Lett. 82, 855 (1999).

[13] I. Sendiña-Nadal, S. Alonso, V. Pérez-Munuzuri, M. GómezGesteira, V. Pérez-Villar, L. Ramírez-Piscina, J. Casademunt, J.M. Sancho, and F. Sagués, Phys. Rev. Lett. 84, 2734 (2000). 
[14] F. Schlögl, C. Escher, and R.S. Berry, Phys. Rev. A 27, 2698 (1983).

[15] A. Rocco, U. Ebert, and W. van Saarloos, Phys. Rev. E 62, R13 (2000).

[16] J.M. Sancho, M. San Miguel, S. Katz, and D. Gunton, Phys. Rev. A 26, 1589 (1982).

[17] L. Ramírez-Piscina and J.M. Sancho, Phys. Rev. A 37, 4469 (1988).

[18] A. Lemarchand, H. Lemarchand, E. Sulpice, and M. Mareschal, Physica A 188, 277 (1992); A. Lemarchand, A. Lesne, and M. Mareschal, Phys. Rev. E 51, 4457 (1995).
[19] W. Horstemke and R. Levefer, Noise-Induced Transitions (Springer, Berlin, 1984).

[20] Miguel A. Santos and J.M. Sancho, Phys. Rev. E 59, 98 (1999).

[21] W. van Saarlos, Phys. Rev. Lett. 58, 2571 (1987); Phys. Rev. A 37, 211 (1989); 39, 6367 (1989).

[22] A. Vasiliev, Yu.M. Romanovskii, D. S. Chernavskii, and V.G. Yakhno, Autowaves Processes in Kinetic Systems (Reidel, Dordrecht, 1987).

[23] A. Rocco, J. Casademunt, and L. Ramírez-Piscina (unpublished). 